\title{
Practical guidance for the implementation of the CRISPR genome editing tool in filamentous fungi
}

\author{
Min Jin Kwon ${ }^{1}$, Tabea Schütze ${ }^{1}$ (D) Sebastian Spohner ${ }^{2}$ (D), Stefan Haefner ${ }^{2}$ and Vera Meyer ${ }^{{ }^{*}}$ (D)
}

\begin{abstract}
Background: Within the last years, numerous reports described successful application of the CRISPR nucleases Cas9 and Cpf1 for genome editing in filamentous fungi. However, still a lot of efforts are invested to develop and improve protocols for the fungus and genes of interest with respect to applicability, scalability and targeting efficiencies. These efforts are often hampered by the fact that-although many different protocols are available - none have systematically analysed and compared different CRISPR nucleases and different application procedures thereof for the efficiency of single- and multiplex-targeting approaches in the same fungus.

Results: We present here data for successful genome editing in the cell factory Thermothelomyces thermophilus, formerly known as Myceliophthora thermophila, using the three different nucleases SpCas9, FnCpf1, AsCpf1 guided to four different gene targets of our interest. These included a polyketide synthase (pks4.2), an alkaline protease (alp 1), a SNARE protein (snc1) and a potential transcription factor (ptf1). For all four genes, guide RNAs were developed which enabled successful single-targeting and multiplex-targeting. CRISPR nucleases were either delivered to T. thermophilus on plasmids or preassembled with in vitro transcribed gRNA to form ribonucleoproteins (RNPs). We also evaluated the efficiency of single oligonucleotides for site-directed mutagenesis. Finally, we were able to scale down the transformation protocol to microtiter plate format which generated high numbers of positive transformants and will thus pave the way for future high-throughput investigations.

Conclusion: We provide here the first comprehensive analysis and evaluation of different CRISPR approaches for a filamentous fungus. All approaches followed enabled successful genome editing in T. thermophilus; however, with different success rates. In addition, we show that the success rate depends on the respective nuclease and on the targeted gene locus. We finally present a practical guidance for experimental considerations aiming to guide the reader for successful implementation of CRISPR technology for other fungi.
\end{abstract}

Keywords: Filamentous fungi, Cell factory, Thermothelomyces thermophilus, Myceliophthora thermophila, CRISPR, Genome editing, Cas9, Cpf1, Cas12a, RNP, Multiplexing, Selection-free gene targeting

\section{Background}

Six million fungal species are estimated to exist on Earth [1], but we know only about 100,000 [2]. Most are saprophytes; however, many pose a threat for other organisms including man. Only a few are exploited in

\footnotetext{
*Correspondence: vera.meyer@tu-berlin.de

${ }^{1}$ Chair of Applied and Molecular Microbiology, Institute of Biotechnology, Technische Universität Berlin, 10263 Berlin, Germany

Full list of author information is available at the end of the article
}

biotechnology as cell factories. Aspergillus niger has been the pioneer fungus of modern biotechnology and used for exactly 100 years for the production of citric acid and since then together with other fungal cell factories for many other products including organic acids, enzymes, drugs, antibiotics and vitamins to name but a few [3, 4]. To improve our understanding on fungal biology underlying pathogenicity or metabolic capabilities, fast and efficient genetic manipulation tools are a fundamental prerequisite. 
The discovery of the CRISPR/Cas9 immune system of bacteria and archaea and their repurposing for genome editing has elicited a new era in genetic engineering for filamentous fungi. Successful applications have been reported for fungal cell factories since 2015 [5] including among others Aspergillus niger, Penicillium chrysogenum, Trichoderma reesei and Thermothelomyces thermophilus, which has recently been reviewed [6-8]. Notably, the Cas12a (Cpf1) system has recently been introduced as an alternative CRISPR tool for $A$. niger [9]. Compared to Cas9, Cpf1 recognizes T-rich PAM sequences and does not need a trans-acting crRNA (tracrRNA) due to the unique dual nuclease activity that cleaves not only the target DNA but also its own CRISPR-RNA (crRNA) [10]. Therefore, it is considered an improved alternative to Cas9 [11]. Currently, various CRISPR protocols have been published for filamentous fungal model strains and cell factories (Table 1), which reflect a broad applicability of this tool but considerably challenge the experimenter to choose the optimal methodology for a specific application or research question. So far, no structured survey has been performed, which systematically analyses and compares the efficiency of different CRISPR nucleases for single- and multiplextargeting in a filamentous fungus and which could provide researchers a guidance outlining the advantages or disadvantages of different CRISPR approaches.

To overcome this issue, we tested in the current study the efficiency of three different nucleases for gene targeting in the cell factory $T$. thermophilus. This filamentous fungus is of current research interest because it exhibits a large capacity for plant biomass degradation and represents a potential reservoir of novel enzymes for many industrial applications. It was formerly known as Myceliophthora thermophila and a CRISPR method based on Cas9 has been published for this cell factory in 2017 [12]. The market for enzymes is huge with a total value of approximately $\$ 4$ billion in 2018 [13]. The market leader was Novozymes, with a share of $48 \%$, followed by Danisco (21\%), DSM
(6\%), AB Enzymes (5\%) and BASF (4\%). Within this market, household care enzymes made up 32\% of sales, closely followed by food and beverage enzymes (29\%), bioenergy (19\%), agricultural and feed (14\%) and other technical and pharma enzymes (6\%). The T. thermophilus strain ATCC 42464 is predominantly used in academic research groups as the general wild-type strain. For industrial use, the proprietary mature enzyme production strain $\mathrm{C} 1$ was developed [14]. The main features of strain $\mathrm{C} 1$ are production levels up to $100 \mathrm{~g} / \mathrm{L}$ protein, and the maintenance of low viscosity levels during fermentation.

We present here a comprehensive survey of different CRISPR gene-targeting approaches for the T. thermophilus strain ATCC 42464 including the successful implementation of two new Cpf1 nucleases. We tested the Cpf1 nucleases from Francisella novicida (FnCpf1) and Acidaminococcus sp. (AsCpf1) to broaden the genome editing toolbox and compared their performance to the well-established Cas9 nuclease from Streptococcus pyogenes (SpCas9). Note that the recognition sequence for FnCpf1 is $5^{\prime}-\mathrm{TTN}-3^{\prime}$ and $5^{\prime}-\mathrm{TTTN}-3^{\prime}$ for AsCpf1, whereas SpCas9 recognizes $5^{\prime}$-NGG-3' ${ }^{\prime}$ [15]. Previous studies have shown that the genome editing efficiency can be different between AsCpf1 and FnCpf1. AsCpf1 performed better in human cell lines [16], whereas genome editing with FnCpf1 was more efficient in S. cerevisiae [17]. Single, double, triple and quadruple genetargeting were successfully established in T. thermophilus and the efficiency of a plasmid-based or RNP-based provision of the respective nucleases compared. We finally optimized transformation protocols for both approaches with respect to efficiency and scalability.

\section{Results and discussion}

RNP application of FnCpf1, AsCpf1 and SpCas9 for single targeting

Strains of filamentous fungi that are deficient in the non-homologous end joining (NHEJ) pathway, i.e. with

Table 1 Filamentous fungal model strains and cell factories for which CRISPR gene editing tools have been established. For details, see reviews [6-8]

\begin{tabular}{|c|c|c|c|}
\hline Species & CRISPR protein & Nuclease delivery & References \\
\hline Aspergillus fumigatus & Cas9 & Plasmid-based and RNP-based & {$[36,37]$} \\
\hline A. nidulans & Cas9, Cpf1 & Plasmid-based & [9] \\
\hline A. niger & Cas9, Cpf1 & Plasmid-based and RNP-based & {$[5,9]$} \\
\hline A. oryzae & Cas9 & Plasmid-based & [38] \\
\hline Neurospora crassa & Cas9 & Plasmid-based & [39] \\
\hline Penicillium chrysogenum & Cas9 & Plasmid- based and RNP-based & [33] \\
\hline Thermothelomyces thermophilus & Cas9 & Plasmid-based & [12] \\
\hline Trichoderma reesei & Cas9 & Plasmid-based & [40] \\
\hline
\end{tabular}

Note that the nuclease can be delivered to the cell either plasmid encoded or as a purified protein, which has to be preassembled with in vitro transcribed gRNA to form ribonucleoproteins (RNPs) 
reduced ectopic integration events during transformation, are preferred as hosts for efficient genome editing due to their high frequencies of DNA integration via homologous recombination [18]. In the case of T. thermophilus, this was recently proven for the ku70 gene, which is a central element of the NHEJ machinery. Its inactivation resulted in a threefold higher homologous recombination rate [12]. Therefore, we have deleted another central element of the NHEJ machinery, the predicted $k u 80$ ortholog, (MYTH_2118116), in the wild-type $T$. thermophilus strain ATCC42464 using SpCas9 and amdS as selection marker (for details see "Methods"). Correct deletion of $k u 80$ was verified in strain MJK19.4 by diagnostic PCR and Southern blot analysis (Additional file 1 and data not shown). This strain was selected for removal of the amdS gene via FAA counterselection (see "Methods") resulting in strain MJK20.2.

In order to compare the three different CRISPR nucleases FnCpf1, AsCpf1 and SpCas9 regarding their targeting efficiency, we chose the $p k s 4.2$ gene and re-used the amdS gene for selection. In the T. thermophilus genome, two orthologs of the pks4 gene described in T. reesei [19] are present, which we named pks4.1 (MYTH_105482) and pks4.2 (MYTH_2300170), respectively. However, only deletion of $p k s 4.2$ results in an easy detectable spore colour change in $T$. thermophilus, thus allowing fast screening of potentially successful CRISPR mutants (Fig. 1a). RNP application, meaning the individual transformation of the respective pre-assembled RNP, of all three CRISPR nucleases achieved 100\% targeting efficiency as confirmed by phenotypic and diagnostic PCR screenings (see "Methods") and increased the transformation efficiency specifically for the FnCpf1 nuclease (Table 2). One has to note, however, that PAM sites are different for Cpf1 (TTN) and Cas9 (NGG); hence, different DNA motifs are targeted in the same gene of interest. This makes a direct comparison of targeting efficiencies difficult, as anything from base pairing of the gRNA, to the target sequence, to chromatin accessibility of the target site can affect this process. Nevertheless, it is assumed that NHEJ-mediated knock-in mediated by the annealing of cohesive ends might be facilitated using Cpf1 proteins, which produce cohesive ends with 4- or 5-nt overhangs, while SpCas9 produces blunt ends [20].

\section{RNP application of FnCpf1, AsCpf1 and SpCas9 for multiplex-targeting}

To investigate whether the three CRISPR nucleases support targeting of up to four genes simultaneously, three of which via a selection-free process, we performed the following strategy: (i) deletion of the pks4.2 gene (using amdS as selection marker) resulting in an easy detectable colour mutant (Fig. 1a), (ii) replacement of the endogenous snc1 gene (MYTH_64173) with a functional snc1::eGFP fusion construct for detection of GFP fluorescence via confocal microscopy (note that $s n c 1$ encodes a SNARE protein and is an established marker for secretory vesicles in filamentous fungi (Fig. 1b, [21]), (iii) deletion of the alp1 gene (MYTH_2303011) encoding an alkaline protease which was previously shown to become
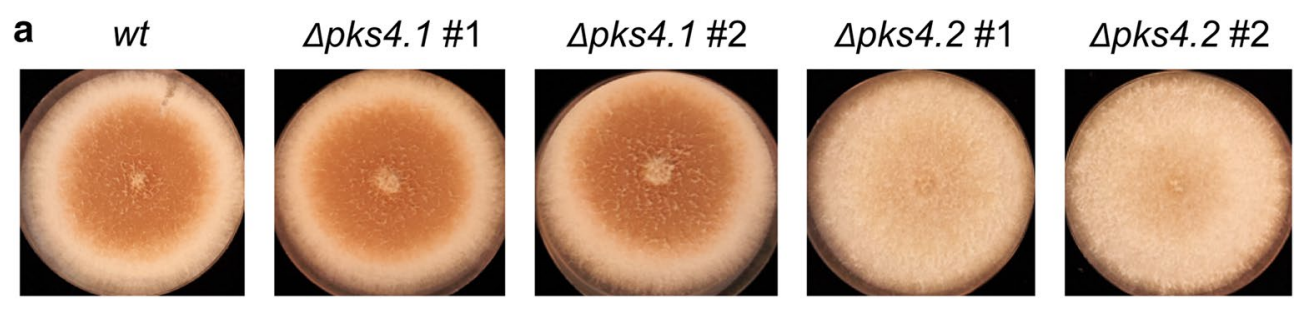

b

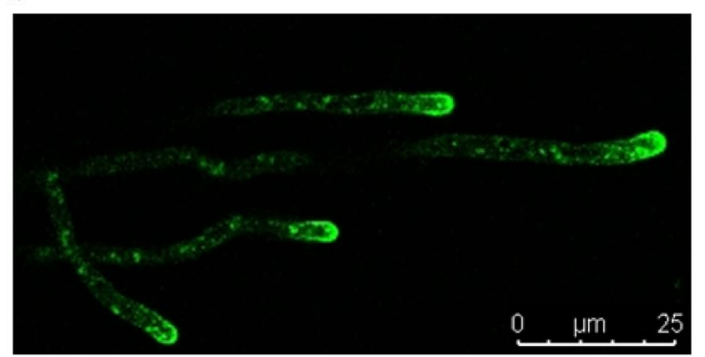

Fig. 1 Phenotypes of T. thermophilus strains generated with CRISPR. a Deletion-phenotype of $p k s 4.1$ and pks4.2 strains cultivated on CM agar plates for 4 days at $37^{\circ} \mathrm{C}$. b GFP::Snc1 strains cultivated on MM agar for $12-16 \mathrm{~h}$ at $28^{\circ} \mathrm{C}$ and analysed via fluorescence microscopy. Secretory vesicles accumulate at the hyphal apex. Note that hyphae of T. thermophilus do not autofluoresce under the experimental condition used (see "Methods", data not shown) 
Table 2 Transformants and gene deletion efficiency targeting the pks4.2 gene

\begin{tabular}{lcc}
\hline Nuclease & No. of colonies analysed & $\begin{array}{l}\text { Editing } \\
\text { efficiency } \\
\text { (\%) }\end{array}$ \\
\hline- & 9 & 25 \\
FnCpf1 & 43 & 100 \\
AsCpf1 & 15 & 100 \\
SpCas9 & 11 & 100 \\
\hline
\end{tabular}

Phenotypic screens and diagnostic PCR for the $p k s 4.2$ locus were performed as described in "Methods" section

Table 3 Transformants and PCR-confirmed editing efficiency for the simultaneously targeted alp1, pks4.2, snc1, and ptf1 gene loci

\begin{tabular}{lccccc}
\hline Nuclease & No. of colonies & \multicolumn{5}{l}{ Editing efficiency (\%) } \\
\cline { 3 - 6 } & & alp1 & pks4.2 & snc1 & ptf1 \\
\hline- & 1 & 0 & 100 & 0 & 0 \\
FnCpf1 & 56 & 91 & 36 & 52 & 6 \\
AsCpf1 & 80 & 92 & 10 & 49 & 0 \\
SpCas9 & 40 & 20 & 97 & 0 & 20 \\
\hline
\end{tabular}

Diagnostic PCR for all individual loci were performed as described in "Methods" section

successfully targeted by SpCas9 in T. thermophilus [12], and (iv) deletion of a non-verified protein encoding a predicted transcription factor. For brevity, we named it $p t f 1$ in this study. Donor DNAs were provided for all genes and details can be found in "Methods" section.

As depicted in Table 3, the targeting efficiencies of FnCpf1 and AsCpf1 are very similar. However, all three nucleases target the four gene loci with different efficiencies. Whereas the $p t f 1$ gene seems to be generally difficult to edit for all three nucleases, the alp1 gene might only be difficult as a target for the SpCas9 nuclease, implying that the performance of CRISPR nucleases can also be locus-dependent. Alternatively, the same gRNA might be recognized with different efficiencies by the different nucleases, as shown in other studies (e.g. [22]). Surprisingly, SpCas9 was not able to target the $s n c 1$ gene during two independent transformation attempts, although the respective in vitro control cleavage assay proved that the enzyme is functional with the gRNA provided (Additional file 2).

Notably, the identification of single, double, triple or quadruple targeting events in T. thermophilus induced by the RNP multiplexing approach uncovered that all nucleases display different abilities to target multiple genes simultaneously, whereby quadruple targeting events were very seldom(Fig. 2). Reduced targeting efficiency is a generally observed phenomenon when multiple genes become simultaneously targeted, as e.g. recently shown for the cell factory A. niger [23]. Interestingly, quadruple targeting events in T. thermophilus were only detectable for FnCpf1. We have therefore selected this nuclease for a comparative analysis with a plasmid-based expression of FnCpf1.

\section{Comparison of RNP-based and plasmid-based application of FnCpf 1 for multiplex-targeting}

For this study, we cloned the FnCpf1 encoding gene into one plasmid (MT2286) and used it for co-transforming it together with plasmids encoding all four gRNAs separated by direct repeats (plasmid pM)K31.1 for $p k s 4.2$ and snc1, plasmid pMJK32.2 for alp1 and plasmid pMJK33.1 for $p k s 4.1$ and $p t f 1$ ) and each respective donor construct for the pks4.2, snc1, alp1 and ptf1 genes into T. thermophilus (see "Methods"). All plasmids ensured transient expression of the fncpf1 gene and the gRNA encoding sequences. This setup was used for direct comparison with RNP-based application of FnCpf1, which was performed in parallel. Note that for these experiments an improved transformation protocol was developed, in which the transformation efficiency was considerably increased by using PEG-4000 instead of PEG-6000 (see "Methods"). As summarized in Table 4, some differences were observed between single targeting when the FnCpf1 nuclease was provided via RNPs or on a plasmid to $T$. thermophilus (alp1 $\mathrm{p}=0.06, p k s 4.2 \mathrm{p}=0.04, p t f 1 \mathrm{p}=0.83$, snc $1 \mathrm{p}=0.02$, $\mathrm{p}$-values were calculated using a two-tailed students $t$ test). However, the efficiency of double, triple and quadruple targeting considerably improved when the fncpf1 was transiently expressed from a plasmid (Fig. 3a). Remarkably, the overall transformation frequency was higher in the plasmid-based approach compared to the RNP approach (Fig. 3b). This data might suggest that plasmids might be easier taken up by protoplasts compared to RNPs and/or that both FnCpf1 and gRNAs might be more abundant or longer available intracellularly when transiently expressed. For both cases, however, the transformation frequency decreased considerably with an increase in target loci (Fig. 3b).

\section{SON-based targeting of FnCpf1 and SpCas9}

Single-stranded oligonucleotides (SONs) have been shown to be efficient templates for the repair of SpCas9 and LbCpf1 (from Lachnospiraceae bacterium) induced DNA double-strand breaks in NHEJ-deficient $A$. nidulans and A. niger [9, 24]. We therefore tested whether this approach (which can be harnessed to introduce specific point mutations into the locus of interest) can also be followed using FnCpf1 and SpCas9 nucleases in T. thermophilus. We thus applied $90 \mathrm{bp}$ long oligonucleotides 


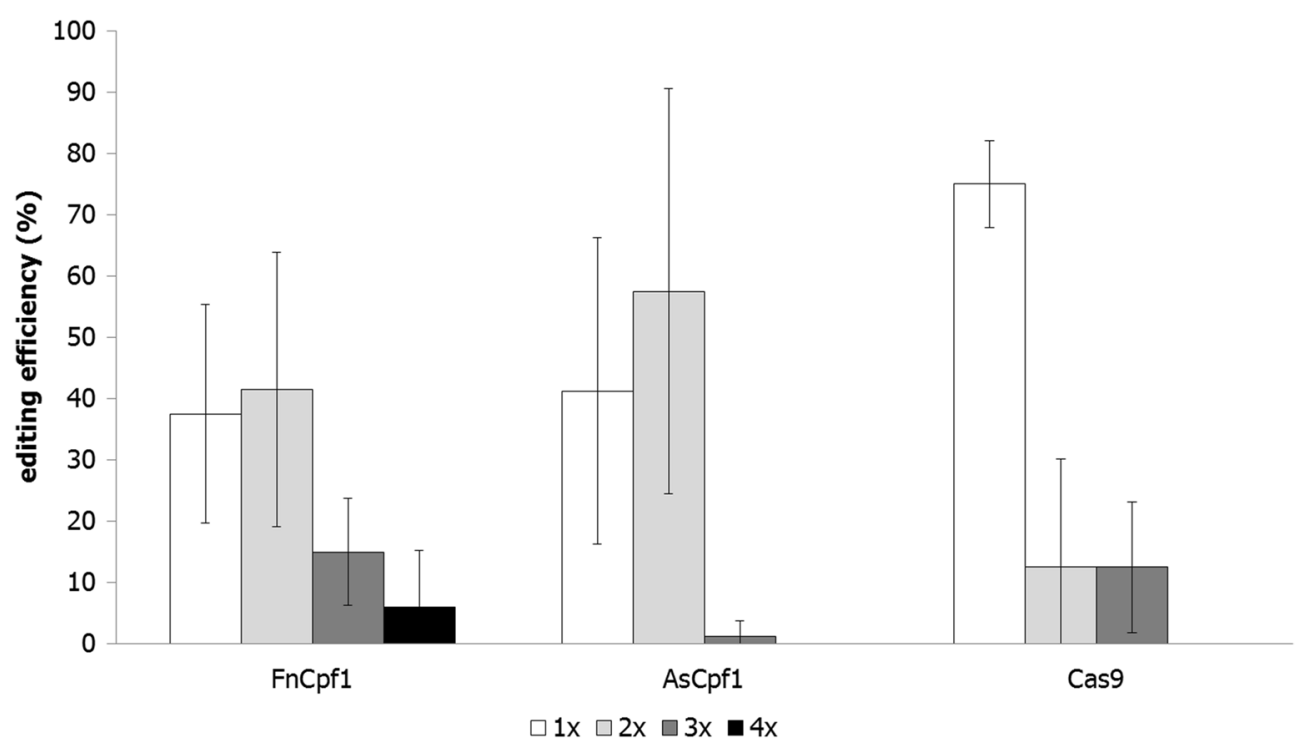

Fig. 2 Editing efficiencies of four targets using the RNP approach. Three different nucleases were used to target 4 genes simultaneously (pks4.2, alp 1, snc1, ptf1). Only the donor DNA of $\Delta p k s 4.2$ contained the selection marker amdS. Up to four individual transformation experiments were performed and 40-80 transformants were analyzed for each setup

Table 4 Editing efficiency for four different gene loci of FnCpf1

\begin{tabular}{|c|c|c|c|c|c|c|c|c|}
\hline \multirow[t]{2}{*}{ Target } & \multicolumn{4}{|l|}{ RNP } & \multicolumn{4}{|l|}{ Plasmid } \\
\hline & Mean [\%] & SD [\%] & $n$ & $\begin{array}{l}\text { No. of analysed } \\
\text { transformants }\end{array}$ & Mean [\%] & SD [\%] & $n$ & $\begin{array}{l}\text { No. of analysed } \\
\text { transformants }\end{array}$ \\
\hline alp1 & 93.9 & 9.4 & 7 & 108 & 80 & 7.1 & 4 & 78 \\
\hline pks4.2 & 50 & 34.3 & 13 & 228 & 100 & 0 & 10 & 158 \\
\hline sncl & 57.2 & 17.7 & 11 & 185 & 40 & 10.4 & 6 & 118 \\
\hline ptfi & 7 & 6.6 & 3 & 28 & 5.5 & 7.8 & 2 & 38 \\
\hline
\end{tabular}

$S D$ standard deviation, $n$ number of transformations performed

Diagnostic PCR was done for alp1 and ptf1 loci as described in "Methods" section. For snc1, fluorescence microscopy and for pks4.2, the colour of conidia was used to verify successful integration of the donor DNA. The donor DNA for pks4.2 was carrying the amdS selection marker

homologous to part of the pks4.2 locus which were designed to introduce three stop codons in the centre part (Additional file 3). The selection marker was present on the donor DNA for the second target, pks4.1 (plasmid pMJK22.19). In total, 30 (25) amdS expressing transformants were identified for FnCpf1 (SpCas9), 5 (3) of which displayed the respective spore colour change indicative for a pks4.2 gene inactivation. All eight transformants were picked and sub-cultivated. Correct integration of the pks4.1 donor DNA was verified by PCR and the respective pks4.2 locus PCR amplified and sequenced. The sequencing results verified that all 8 transformants were successfully targeted by both nucleases and that the SON introduced the desired gene edits (Additional file 3). For the first time, this data provides evidence that an oligonucleotide-mediated repair approach can be followed in T. thermophilus for site-directed mutagenesis applying either FnCpf1 or SpCas9.

\section{MTP-based method for high-throughput gene targeting}

We finally provide here a protocol for high-throughput gene targeting in T. thermophilus using a microtiter plate (MTP) compatible method. MTP-based approaches are fundamental for the development of cost-effective workflows used for genome-wide mutant libraries and highthroughput screenings for protein production. Recently, a respective protocol has been reported for A. niger [25], however, none has been published for $T$. thermophilus so far. Figure 4 summarizes the key aspects of this down-scaled approach, where $10 \mu \mathrm{L}$ of protoplast solution $\left(\sim 5 \times 10^{5}\right.$ protoplasts) are sufficient to obtain $>30$ transformants with either an RNP- or plasmid-based 

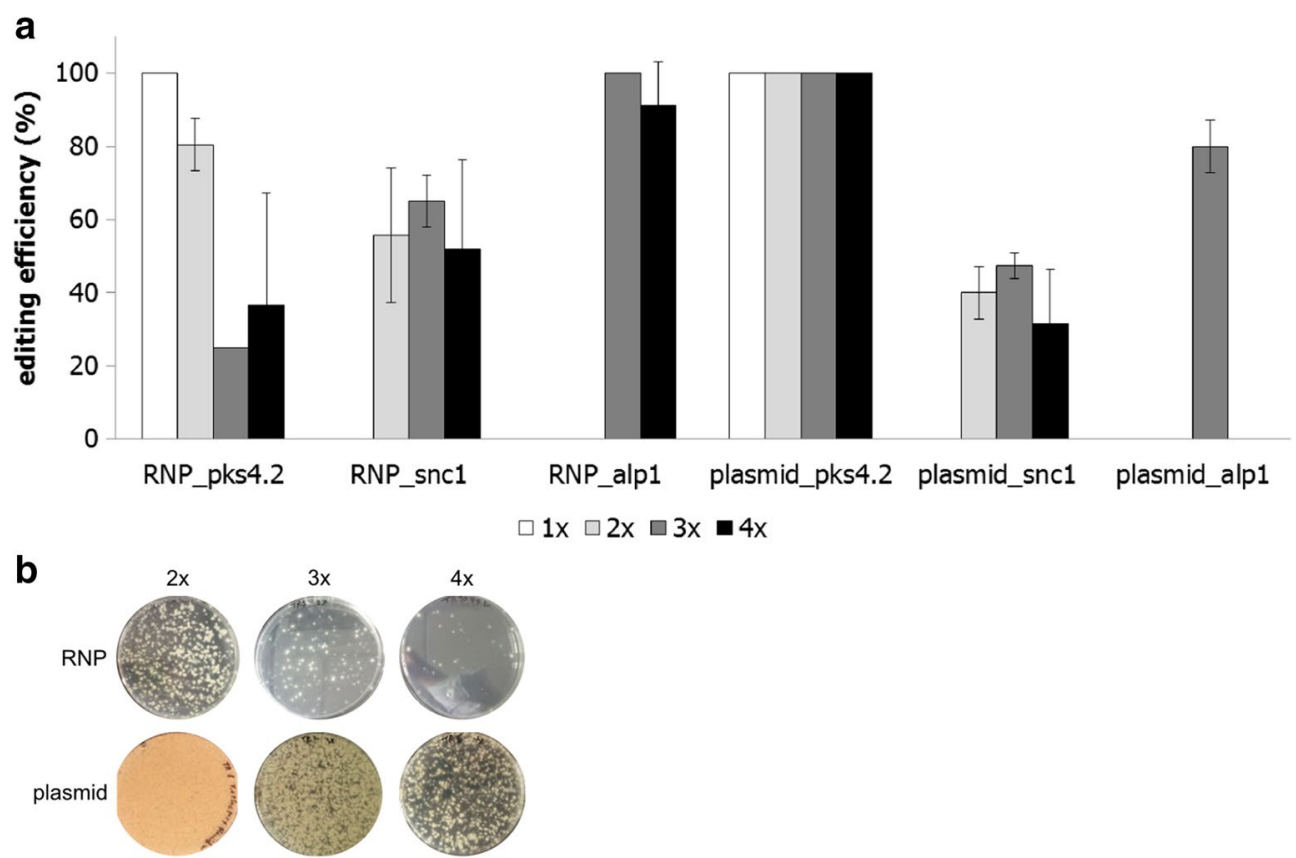

Fig. 3 Comparison of the RNP- and plasmid-based approach. a Editing efficiencies for single, double, triple and quadruple targeting. FnCpf1 was used to target all 4 genes simultaneously (' $4 x^{\prime}$ ' $p k s 4.2$, alp1, sncl, ptfl) or only double or triple combinations thereof. Up to ten individual transformation experiments were performed for each setup. Note that no experiments for RNP_snc1 (1X), RNP_alp1 (1x) and $(2 \times)$, plasmid_snc1 $(1 \times)$, plasmid_alp1 $(1 \times),(2 x)$ and $(4 x)$ were done. Data for ptf1 were omitted in the diagram due to low targeting efficiency. $\mathbf{b}$ Pictures of transformation plates using different amounts of targets. Note that the targets for the RNP- and plasmid-based approach were identical except for $4 \times$ targeting. There, the targets were alp 1, pks4.2, sncl, and ptf1 for the RNP approach and alp 1, pks4.1, pks4.2 and sncl for the plasmid approach

approach of FnCpf1 targeting the genes pks4.2 and/or snc1, respectively. As with the classical transformation protocol, the single targeting efficiency was $100 \%$ (tested for $p k s 4.2$ only) and the double targeting efficiency was $40-56 \%$ (tested for $p k s 4.2$ and $s n c 1$ ). Similarly, plasmidbased application of FnCpf1 yielded more transformants than the RNP-based application on MTP scale. An MTPbased workflow is thus indeed very promising for highthroughput approaches. In this context, it is interesting to note that the addition of the cryoprotectant polyvinylpyrrolidone 40 to freshly harvested protoplasts from T. thermophilus allowed us to store protoplasts at $-80^{\circ} \mathrm{C}$ for several weeks prior to transformation with no significant reduction in viability and transformability (see "Methods", data not shown).

\section{Conclusions}

CRISPR applications in fungal systems are on exponential rise with the same pace as in other biological systems and drive new research for medically and industrially relevant filamentous fungi [26]. Dozens of CRISPR articles have been published for filamentous fungi including many reviews since the first report on CRISPR-Cas9 application in Aspergilli in 2015 [5]. The huge scientific interest in the community to explore fungal diversity and specification even within a single genus $[27,28]$ will eventually lead to more filamentous fungi of interest for which efficient genetic transformation and genome editing tools have to be developed. The work presented here systematically investigated and evaluated different experimental approaches for the cell factory $T$. thermophilus, most of which were not tested yet for this fungus. We could successfully establish a variety of scalable protocols enabling genome editing by three different CRISPR nucleases for single- and multiplex-targeting approaches using plasmid-based or RNP-based delivery of the enzymes. We furthermore provide evidences that SON-mediated mutagenesis is functional in T. thermophilus for two CRISPR nucleases. Table 5 summarizes opportunities, limitations and critical aspects related to practicability and efficiency based on our observations made for T. thermophilus. It also reflects what has been observed so far in other eukaryotic systems (see references cited above). It could thus guide fungal scientists for future implementation of the CRISPR technology for their filamentous fungus of interest. In general, the availability of different Cas nucleases is of advantage. In case one enzyme fails to target a gene of interest, it is likely 


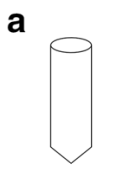

$50 \mathrm{ml}$ tube

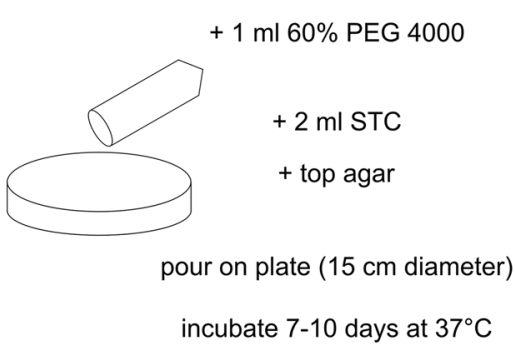

b

\begin{tabular}{|c|c|c|c|c|c|c|}
\hline approach/target(s) & & RNP & & & plasmid & \\
\hline & mean $[\%]$ & $\begin{array}{l}\text { analysed } \\
\text { transformants }\end{array}$ & $\mathrm{n}$ & mean $[\%]$ & $\begin{array}{l}\text { analysed } \\
\text { transformants }\end{array}$ & $n$ \\
\hline$p k s 4.2$ & 100 & 10 & 2 & 100 & 10 & 4 \\
\hline \multirow[t]{2}{*}{$p k s 4.2 \&$ snc1 } & - & - & - & $100 \& 40$ & 15 & 2 \\
\hline & & & & $100 \& 56$ & 16 & \\
\hline
\end{tabular}

$10 \mu$ protoplasts

$1 \mu$ d donor DNA $(\sim 1 \mu \mathrm{g})$

$2 \mu \mathrm{l}$ FnCpf1, gRNA plasmid DNA $(\sim 1 \mu \mathrm{g})$

or $5 \mu$ l of RNP mixture

$2.5 \mu l 60 \%$ PEG

$1.5 \mathrm{ml}$ tube

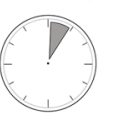

$+61.5 \mu$ l 60\% PEG 4000

$+123 \mu$ STC

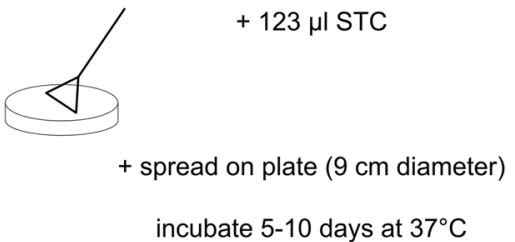

C

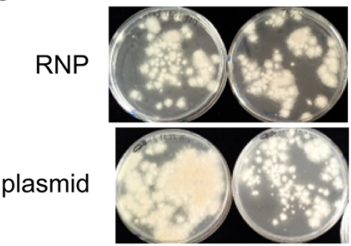

Fig. 4 Downscaling the transformation procedure. a Comparison of medium- and small-scale transformation, details on the methodology can be found in "Methods" section. b Table comparing the genome editing efficiency of small-scale transformation between the RNP- and plasmid-based approach, $n=$ number of transformations performed. $\mathbf{c}$ Backside of four transformation plates from small-scale transformations are shown, which demonstrate that a reduced number of transformants will be gained with the small-scale protocol when compared to the medium-scale protocol (see Fig. 3b)

that another will do. The approach which is followed to deliver the Cas nuclease will also affect editing efficiencies. Be it that a plasmid-based approach might be easier to handle and is less prone to degradation compared to a RNP-based approach, be it because transient expression from plasmids (which are presumably longer stable intracellularly compared to RNPs) might ensure higher abundance and longer availability of the Cas enzymes and their gRNAs. It can also be speculated that targeting with RNPs (especially when multiplexing is intended) might exceed the limit of DNA/RNA which can be taken up by protoplasts. The canonical view is that an excess of DNA lowers transformation efficiency in filamentous fungi. Another important aspect worth considering, especially when using SpCas9, is the potential of introducing unintended off-target mutations [20]. The chances for hitting potential off-targets is likely high when both integration of the Cas9 nuclease in the genome and its constitutive expression is performed, lower when transient expression is adjusted but should be lowest, when an RNP-based approach is followed.

\section{Methods}

\section{Microbial strains and cultivation conditions}

Fungal strains used in this study are given in Additional file 4. Strain MJK20.2 was used as progenitor isolate as this strain is deficient in the non-homologous end joining pathway $(\Delta k u 80)$, reducing ectopic integration events during transformation and thus enabling targeted integration [29]. Strains were grown at $37{ }^{\circ} \mathrm{C}$ in minimal medium (MM) or complete medium $(\mathrm{CM})$, consisting of MM supplemented with $1 \%$ yeast extract and $0.5 \%$ casamino acids [18]. All bacterial plasmids were propagated in Escherichia coli DH5 $\alpha$ using $100 \mu \mathrm{g} / \mathrm{mL}$ ampicillin or $50 \mu \mathrm{g} / \mathrm{mL}$ kanamycin for selection.

\section{Molecular techniques}

All molecular techniques were performed according to standard procedures described previously [30]. T. thermophilus transformation and genomic DNA extraction were performed as described elsewhere [18]. When required, plates were supplemented with acetamide $(15 \mathrm{mM})$ and caesium chloride $(10 \mathrm{mM})$. Primers and plasmids used in this study are given in Additional files 5 and 6, respectively. All plasmids were sequenced and will 
Table 5 Practical guidance for the implementation of CRISPR technology in filamentous fungi based on data obtained for T. thermophilus in this study

\begin{tabular}{|c|c|c|}
\hline & Plasmid-based approach & RNP-based approach \\
\hline Preparation of nuclease & $\begin{array}{l}\text { Cloning of the nuclease into a plasmid prior } \\
\text { transformation is mandatory. When constitutively } \\
\text { expressed, risk of off-targets should be considered. } \\
\text { When present on AMA-plasmid, the risk should be } \\
\text { lower but still present }\end{array}$ & $\begin{array}{l}\text { Cloning of the nuclease into a plasmid allowing het- } \\
\text { erologous expression, e.g. in E. coli, is a prerequisite. } \\
\text { Once established and purified, the nuclease can be } \\
\text { aliquoted and stored prior to use. As the protein } \\
\text { does not become expressed in the targeted fungus, } \\
\text { the risk of off-targets should be very small }\end{array}$ \\
\hline Preparation of guide RNA & $\begin{array}{l}\text { Plasmid-based, thus more stable during handling } \\
\text { and storage }\end{array}$ & $\begin{array}{l}\text { Involves in vitro transcription, hence potentially sensi- } \\
\text { tive to handling errors }\end{array}$ \\
\hline Transformation procedure & Easy & Easy but requires preassembly of RNPs \\
\hline Transformation rate & Very high also with four targets & $\begin{array}{l}\text { Very high for single and double targets } \\
\text { Low for three and four targets }\end{array}$ \\
\hline $\begin{array}{l}\text { Single-targeting efficiency of FnCpf1, } \\
\text { AsCpf1, SpCas9 }\end{array}$ & Locus-dependent & Locus-dependent \\
\hline Multiplex-targeting efficiency of FnCpf1 & High (34\% $\pm 6 \%$ in this study) & Low $(13 \% \pm 2 \%$ in this study) \\
\hline MTP-based down-scaling for FnCpf1 & $\begin{array}{l}\text { Possible with no loss in efficiency with respect to } \\
\text { single and double targeting }\end{array}$ & $\begin{array}{l}\text { Possible with no loss in efficiency with respect to } \\
\text { single targeting* }\end{array}$ \\
\hline
\end{tabular}

*Double targeting was not tested

be made available on reasonable request. Strain MJK20.2 $(\Delta k u 80)$ was generated as follows: $k u 80$ was deleted in the wild type strain ATCC42464 with FnCpf1 or SpCas9 using PCR-amplified split marker fragments containing the amdS marker and about $1.2 \mathrm{~kb}$ flanks each for homologous integration. The $3^{\prime}$ split marker fragment contained the $5^{\prime}$ flank to mediate a fast removal of the amdS marker. The resulting strain MJK19.1 was subcultivated on FAA medium plates to obtain the markerfree $\Delta k u 80$ strain MJK20.2. Strains were analysed by Southern blot analysis to verify correct integration of the fragments and removal of the marker gene (Additional file 1). For all other targets, donor DNA with flanks of about $1 \mathrm{~kb}$ length each were used. The amounts of donor DNA are specified in the RNP-based and plasmid-based approaches described below.

\section{Genome editing using RNP-based approach}

The plasmid containing the expression cassette for SpCas9 (pET28a/Cas9-Cys) was obtained from addgene (\#53261). T. thermophilus codon optimized FnCpf1 and AsCpf1 was cloned into plasmid pET28a giving plasmid pMJK16.1 and pMJK17.1, respectively. E. coli strain Rosetta $^{\mathrm{TM}}$ 2(DE3)pLysS (Novagen) was freshly transformed with the respective expression plasmids. Four $\mathrm{mL}$ of TB medium (12 g/L tryptone, $24 \mathrm{~g} / \mathrm{L}$ yeast extract, 5 g/L glycerol, $2.31 \mathrm{~g} / \mathrm{L} \mathrm{KH}_{2} \mathrm{PO}_{4}, 12.54 \mathrm{~g} / \mathrm{L} \mathrm{K}_{2} \mathrm{HPO}_{4}$ ) plus $50 \mu \mathrm{g} / \mathrm{mL}$ kanamycin and $20 \mu \mathrm{g} / \mathrm{mL}$ chloramphenicol were inoculated from a single colony and incubated at $37{ }^{\circ} \mathrm{C}$ and $250 \mathrm{rpm}$ overnight. $400 \mu \mathrm{L}$ of these precultures were used to inoculate $40 \mathrm{~mL}$ of $\mathrm{TB}$ medium including antibiotics, which was incubated at $37{ }^{\circ} \mathrm{C}$ and $250 \mathrm{rpm}$ until an optical density $\left(\mathrm{OD}_{600}\right)$ of 5.0-8.0 was reached (approximately 5-7 h). Main cultures (1 L in 5 L Erlenmeyer flasks) with TB medium, autoinduction solution (5 g/L glycerol, $0.5 \mathrm{~g} / \mathrm{L}$ glucose, $2 \mathrm{~g} / \mathrm{L} \alpha$-lactose monohydrate) and the corresponding antibiotic(s) were inoculated with these $40 \mathrm{~mL}$ cultures to an $\mathrm{OD}_{600}$ of 0.1 and incubated in shake flasks at $37{ }^{\circ} \mathrm{C}$ and $160 \mathrm{rpm}$ for $2 \mathrm{~h}$. Afterwards, the temperature was decreased to $18{ }^{\circ} \mathrm{C}$ and the cells cultivated for at least 18 up to a maximum of $40 \mathrm{~h}$. Proteins were purified as described previously [31] using Ni-NTA resin (Qiagen Germany).

Target sequences were selected in silico using CasDesigner and Cas-OFFinder (http://www.rgenome.net/ cas-offinder/) [32]. Respective gRNAs including PAM sites were generated as described before [33]. In brief, gRNAs were in vitro transcribed using the T7 promoter with an additional ATG at the front (ATGTAATACGAC TCACTATAGG). For sequence information, see Additional file 5 .

RNP assembly was done as described previously [33] with the following modifications. Prior to the transformation into fungal protoplasts, RNP complexes were assembled containing $30 \mu \mathrm{g}$ CRISPR nuclease $(5 \mu \mathrm{L})$, $2 \mu \mathrm{L} 10 \times$ Cas 9 activity buffer, $1 \mu \mathrm{L}$ gRNA and $12 \mu \mathrm{L}$ of nuclease-free water in a $1.5 \mathrm{~mL}$ reaction tube. The mixture was incubated at $37{ }^{\circ} \mathrm{C}$ for $15 \mathrm{~min}$ to allow RNP complex formation. For multiplex-targeting, each target RNP complex was formed separately. For each transformation, $100 \mu \mathrm{L}$ protoplasts, $10 \mu \mathrm{L}$ donor DNA $(5 \mu \mathrm{g})$, $20 \mu \mathrm{L}$ RNP complex (up to $80 \mu \mathrm{L}$ for multiplex-targeting), $20 \mu \mathrm{L} 2 \times$ STC, $25 \mu \mathrm{L}$ 60\% PEG 4000 buffer and $20 \mu \mathrm{L}$ $10 \times$ Cas 9 activity buffer were mixed in a $50 \mathrm{~mL}$ Greiner 
tube. Transformations with plasmid MT28 and/or sterile water served as controls. Note that this protocol differed from [18] with respect to PEG: 60\% PEG 4000 was used in this study instead of 25\% PEG 6000. Transformants were sub-cultivated twice on medium with $15 \mathrm{mM}$ acetamide as nitrogen source. Genomic DNA was extracted from transformants. Insertion of the donor cassette at the respective locus was confirmed by diagnostic PCR.

\section{Genome editing using plasmid-based approach}

$3 \mu \mathrm{g}$ of the fncpf1 encoding plasmid MT2286 was cotransformed with $2 \mu \mathrm{g}$ of each plasmid DNA encoding respective gRNAs separated by direct repeats (e.g. pMJK31.1 for pks4.2 \& snc1 gRNA) as described by [34] and $3 \mu \mathrm{g}$ donor DNA into T. thermophilus as follows: $100 \mu \mathrm{L}$ protoplasts $\left(\sim 5 \times 10^{6}\right.$ protoplasts $), 10 \mu \mathrm{L}$ total DNA and $25 \mu \mathrm{L} 60 \%$ PEG 4000 buffer were mixed in a $50 \mathrm{~mL}$ Greiner tube at room temperature. For transcription of the gRNA the U6 promoter was used. Expression of $f n c p f 1$ was done according to [34, 12]. For codon optimisation the most frequent codons were used [35]. Transformations with plasmid MT28 and/or sterile water served as controls. Note that this protocol differed from [18] with respect to PEG: 60\% PEG 4000 was used in this study instead of 25\% PEG 6000. Transformants were sub-cultivated twice on medium with $15 \mathrm{mM}$ acetamide as nitrogen source. Genomic DNA was extracted from putative transformants. Insertion of the donor cassette at the respective locus was confirmed by diagnostic PCR.

\section{Genome editing using SON-based approach}

SON-based donor DNA targeting $p k s 4.2$ was designed with 35/32 bp (up-/downstream) homologous arms containing 3 stop codons. For sequence information, see Additional file 3. Selection was based on the $\Delta p k s 4.1$ deletion cassette (pMJK22.19), hence a double targeting approach was followed: RNP complexes were assembled containing $30 \mu \mathrm{g}$ FnCpf1 $(5 \mu \mathrm{L}), 2 \mu \mathrm{L} 10 \times$ Cas9 activity buffer, $1 \mu \mathrm{l}$ gRNA and $12 \mu \mathrm{L}$ of nuclease-free water in a $1.5 \mathrm{~mL}$ reaction tube. The mixture was incubated at $37^{\circ} \mathrm{C}$ for $15 \mathrm{~min}$ to allow RNP complex formation. For each transformation, $100 \mu \mathrm{L}$ protoplasts, $5 \mu \mathrm{L}$ donor DNA $(5 \mu \mathrm{g}), 10 \mu \mathrm{L}$ SON (100 $\mu \mathrm{M}$ stock solution), $40 \mu \mathrm{L}$ RNP complex, $20 \mu \mathrm{L} 2 \times$ STC, $25 \mu \mathrm{L}$ 60\% PEG 4000 buffer and $20 \mu \mathrm{L} 10 \times$ Cas 9 activity buffer were mixed in a $50 \mathrm{~mL}$ Greiner tube. Note that this protocol differed from [18] with respect to PEG: 60\% PEG 4000 was used in this study instead of 25\% PEG 6000. Transformants were sub-cultivated twice on medium with $15 \mathrm{mM}$ acetamide as nitrogen source. Genomic DNA was extracted from transformants. Insertion of the donor cassette at the respective locus was confirmed by diagnostic PCR.

\section{Genome editing using MTP-based approach}

The volume for the transformation reaction was reduced to $200 \mu \mathrm{L}$ and transformation was done in a $1.5 \mathrm{~mL}$ reaction tube. Both, freshly prepared and cryopreserved protoplasts have been used. For one transformation using the plasmid approach, $10 \mu \mathrm{L}$ protoplasts $\left(\sim 5 \times 10^{5}\right)$ were mixed with $1 \mu \mathrm{L}$ donor DNA $(1 \mu \mathrm{g}), 1 \mu \mathrm{L}$ FnCpf1 $(1 \mu \mathrm{g})$, $1 \mu \mathrm{L}$ gRNA plasmid $(1 \mu \mathrm{g})$ and $2.5 \mu \mathrm{L} 60 \%$ PEG 4000 buffer at room temperature. Afterwards $61.5 \mu \mathrm{L} 60 \%$ PEG 4000 buffer was added and exactly five minutes later $123 \mu \mathrm{L}$ STC was added. Instead of using top agar to distribute cells on an agar plate ( $15 \mathrm{~cm}$ diameter), the $200 \mu \mathrm{L}$ protoplast mix was spread onto a small plate $(9 \mathrm{~cm}$ diameter). For the RNP approach, $5 \mu \mathrm{L}$ of RNP mixture was added from a $20 \mu \mathrm{L}$ RNP complex reaction mixture. Identification and analysis of transformants were performed as described above.

\section{Cryopreservation of protoplasts}

350-500 $\mu \mathrm{L}$ protoplasts $\left(\sim 1 \times 10^{7}\right)$ were mixed $1: 1$ with $20 \%$ Polyvinylpyrrolidone 40 solved in STC buffer. This mixture was deep frozen at $-80{ }^{\circ} \mathrm{C}$ using isopropanol for $-1{ }^{\circ} \mathrm{C} / \mathrm{min}$ freezing. Before transformation, frozen protoplasts were washed with $10 \mathrm{~mL}$ cold STC buffer and spun down for $5 \mathrm{~min}$ at $1500 \mathrm{rpm}$ and $4{ }^{\circ} \mathrm{C}$. Protoplasts were resuspended with cold STC and used for transformation.

\section{In vitro SpCas9 cleavage assay}

In brief, the plasmids with either the original Psnc1::gfp::snc1 sequence or the SpCas9 PAM site mutated sequence were used as donor DNAs. Each $600 \mathrm{ng}$ were restricted with $10 \mathrm{U}$ NotI in a total volume of $20 \mu \mathrm{L}$ to generated linearized DNAs. After heatinactivation $\left(20 \mathrm{~min}\right.$ at $\left.80^{\circ} \mathrm{C}\right)$ of $\mathrm{Not} \mathrm{I}$, the mixtures were immediately added without further purification to a $30 \mu \mathrm{L}$ reaction mixture containing $1 \mu \mathrm{L}$ gRNA and $1 \mu \mathrm{L}$ Cas9 protein. After $60 \mathrm{~min}$ incubation at $37{ }^{\circ} \mathrm{C}$, the reaction was quenched by adding $3 \mu \mathrm{L} 0.5 \mathrm{M}$ EDTA and $7 \mu \mathrm{L}$ $6 \times$ gel loading dye. Samples were incubated for $15 \mathrm{~min}$ at $65{ }^{\circ} \mathrm{C}$ and analysed by electrophoresis on a $1 \%$ agarose gel.

\section{Genotypic, phenotypic and microscopic screens of CRISPR transformants on agar media}

Putative transformants were analysed as follows: $\Delta p k s 4.2$ transformants were sub-cultivated three times on MM agar medium and the spore colour formation compared to the wild-type strain. In case of questionable 
phenotypes, strains were further subjected to diagnostic PCR. Integration of Psnc1::gfp::snc1 was analysed using fluorescence microscopy. In brief, colonies cultivated on selective agar MM medium containing $15 \mathrm{mM}$ acetamide for $24 \mathrm{~h}$ at $37{ }^{\circ} \mathrm{C}$ and fluorescence images were taken using an inverted TCS SP8 (Leica, Germany) as described earlier [30]. Most colonies with GFP-secretory vesicle signals had correct integration of the donor DNA at the snc1 locus as checked by diagnostic PCR ( 99\%). Consequently, transformants with GFP- secretory vesicle signals, were considered $s n c 1$ targeted. For $\Delta p k s 4.1, \Delta a l p 1$ and $\Delta p t f 1$ diagnostic PCR was done on the corresponding locus. For primer sequence information, see Additional file 5 .

\section{Supplementary information}

Supplementary information accompanies this paper at https://doi. org/10.1186/s40694-019-0079-4.

Additional file 1. Verification of ku80 deletion and marker removal. Additional file 2. In vitro cleavage assay of sncl target site for SpCas9.

Additional file 3. Application of SNOs using Cpf1 and Cas9.

Additional file 4. Strains used in this study.

Additional file 5. Primers and gRNA sequences used in this study.

Additional file 6. Plasmids used in this study.

\section{Abbreviations}

CM: Complete medium; Cas: CRISPR-associated proteins; Cpf1: CRISPR from Prevotella and Francisella; CRISPR: Clustered Regularly Interspaced Short Palindromic Repeats; MM: Minimal medium; MTP: Microtiter plate; RNP: Ribonucleoprotein; SON: single-stranded oligonucleotide.

\section{Acknowledgements}

We acknowledge support by the German Research Foundation and the Open Access Publication Funds of TU Berlin. We thank BASF SE for financial support.

\section{Authors' contributions}

MJK designed gene editing primers, generated und purified CRISPR enzyme variants, generated T. thermophilus mutants, and conducted PCR, Southern, phenotypic and microscopic analyses. SS and SH provided the constructs for genome editing using the plasmid-based approach. VM initiated this study, coordinated the project and co-wrote the final text. MJK, TS, SS and SH were involved in discussions and contributed to writing the manuscript. All authors read and approved the final manuscript.

\section{Funding}

Not applicable.

\section{Availability of data and materials}

The data sets, strains used and/or analysed during the current study, and sequences are available from the corresponding authors on reasonable request.

\section{Ethics approval and consent to participate}

Not applicable.

\section{Consent for publication}

Not applicable.

\section{Competing interests}

The authors declare they have no competing interests.

\section{Author details}

${ }^{1}$ Chair of Applied and Molecular Microbiology, Institute of Biotechnology, Technische Universität Berlin, 10263 Berlin, Germany. ${ }^{2}$ BASF SE,

Carl-Bosch-Strasse 38, 67056 Ludwigshafen, Germany.

Received: 12 August 2019 Accepted: 5 October 2019

Published online: 17 October 2019

\section{Bibliography}

1. Taylor DL, Hollingsworth TN, McFarland JW, Lennon NJ, Nusbaum C, Ruess RW. A first comprehensive census of fungi in soil reveals both hyperdiversity and fine-scale niche partitioning. Ecol Monogr. 2014;84:3-20.

2. Helen A. Ainsworth and Bisby's dictionary of the fungi (10th edition). Ref Rev. 2009;23:42

3. Cairns TC, Nai C, Meyer V. How a fungus shapes biotechnology: 100 years of Aspergillus niger research. Fungal Biol Biotechnol. 2018;5:1-14.

4. Meyer V, Andersen MR, Brakhage AA, Braus GH, Caddick MX, Cairns CT, et al. Current challenges of research on filamentous fungi in relation to human welfare and a sustainable bio-economy: a white paper. Fungal Biol Biotechnol. 2016:3:1-17.

5. Nodvig CS, Nielsen JB, Kogle ME, Mortensen UH. A CRISPR-Cas9 system for genetic engineering of filamentous fungi. PLoS One. 2015;10:e0133085.

6. Deng H, Gao R, Liao X, Cai Y. CRISPR system in filamentous fungi: current achievements and future directions. Gene. 2017;627:212-21.

7. Wang H, La Russa M, Qi LS. CRISPR/Cas9 in genome editing and beyond. Annu Rev Biochem. 2016:85:227-64.

8. Song R, Zhai Q, Sun L, Huang E, Zhang Y, Zhu Y, et al. CRISPR/Cas9 genome editing technology in filamentous fungi: progress and perspective. Appl Microbiol Biotechnol. 2019;103(17):6919-32.

9. Vanegas KG, Jarczynska ZD, Strucko T, Mortensen UH. Cpf1 enables fast and efficient genome editing in Aspergilli. Fungal Biol Biotechnol. 2019;6:1-10.

10. Makarova KS, Zhang F, Koonin EV, Institutes N. SnapShot: class 2 CRISPRCas systems. Cell. 2017;168:328-328.

11. Zetsche B, Heidenreich M, Mohanraju P, Fedorova I, Kneppers J, Degennaro EM, et al. Multiplex gene editing by CRISPR — Cpf1 using a single crRNA array. Nat Biotechnol. 2017;35(1):31.

12. Liu Q, Gao R, Li J, Lin L, Zhao J, Sun W, et al. Development of a genomeediting CRISPR/Cas9 system in thermophilic fungal Myceliophthora species and its application to hyper-cellulase production strain engineering. Biotechnol Biofuels. 2017;10:1.

13. Winter AB, Steel AL. The Novozymes Report 2018. https://report2018 novozymes.com.

14. Visser H, Joosten V, Punt PJ, Gusakov AV, Olson PT, Joosten R, et al. Development of a mature fungal technology and production platform for industrial enzymes based on a Myceliophthora thermophila isolate, previously known as Chrysosporium lucknowense C1. Ind Biotechnol. 2011;7:214-23

15. Kim HK, Song M, Lee J, Menon AV, Jung S, Kang YM, et al. In vivo high-throughput profiling of CRISPR-Cpf1 activity. Nat Methods. 2017:14(2):153.

16. Zetsche B, Gootenberg JS, Abudayyeh OO, Slaymaker IM, Makarova KS, Essletzbichler P, et al. Cpf1 is a single RNA-guided endonuclease of a class 2 CRISPR-Cas system. Cell. 2018;163:759-71.

17. Verwaal R, Buiting-Wiessenhaan N, Dalhuijsen S, Roubos JA. CRISPR/Cpf1 enables fast and simple genome editing of Saccharomyces cerevisiae. Yeast. 2018;35:201-11.

18. Arentshorst M, Ram AFJ, Meyer V. Using non-homologous end-joiningdeficient strains for functional gene analyses in filamentous fungi. Methods Mol Biol. 2012:835:133-50.

19. Atanasova L, Knox BP, Kubicek CP, Druzhinina IS, Baker E. The polyketide synthase gene pks 4 of Trichoderma reesei provides pigmentation and stress resistance. Eukaryotic cell. 2013;12:1499-508.

20. Nakade S, Yamamoto T, Sakuma T. Cas9, Cpf1 and C2c1/2/3-What's next? Bioengineered. 2013;4(3):265-73.

21. Kwon MJ, Nitsche BM, Arentshorst M, Jørgensen TR, Ram AFJ, Meyer V. The transcriptomic signature of RacA activation and inactivation provides new insights into the morphogenetic network of Aspergillus niger. PLoS ONE. 2013;8(7):e68946. 
22. Zhang J, Yang F, Yang Y, Jiang Y, Huo Y-X. Optimizing a CRISPR-Cpf1-based genome engineering system for Corynebacterium glutamicum. Microb Cell Fact. 2019;18:60.

23. van Leeuwe TM, Arentshorst M, Ernst T, Alazi E, Punt PJ, Ram AFJ. Efficient marker free CRISPR/Cas9 genome editing for functional analysis of gene families in filamentous fungi. Fungal Biol Biotechnol. 2019;6:13.

24. Nødvig CS, Hoof JB, Kogle ME, Jarczynska ZD, Lehmbeck J, Klitgaard DK, et al. Efficient oligo nucleotide mediated CRISPR-Cas9 gene editing in Aspergilli. Fungal Genet Biol. 2018;115:78-89.

25. Kuivanen J, Korja V, Holmström S, Richard P. Development of microtiter plate scale CRISPR/Cas9 transformation method for Aspergillus niger based on in vitro assembled ribonucleoprotein complexes. Fungal Biol Biotechnol. 2019:6:1-12.

26. Idnurm A, Meyer $\mathrm{V}$. The CRISPR revolution in fungal biology and biotechnology, and beyond. Fungal Biol Biotechnol. 2018;5:19.

27. de Vries RP, Riley R, Wiebenga A, Aguilar-Osorio G, Amillis S, Uchima CA, et al. Comparative genomics reveals high biological diversity and specific adaptations in the industrially and medically important fungal genus Aspergillus. Genome Biol. 2017;18:28.

28. Vesth TC, Nybo JL, Theobald S, Frisvad JC, Larsen TO, Nielsen KF, et al. Investigation of inter- and intraspecies variation through genome sequencing of Aspergillus section Nigri. Nat Genet. 2018;50:1688.

29. Meyer V, Arentshorst M, El-Ghezal A, Drews A-C, Kooistra R, den Hondel CA, et al. Highly efficient gene targeting in the Aspergillus niger kusA mutant. J Biotechnol. 2007;128:770-5.

30. Fiedler MRM, Cairns TC, Koch O, Kubisch C, Meyer V. Conditional expression of the small GTPase ArfA impacts secretion, morphology, growth, and actin ring position in Aspergillus niger. Front Microbiol. 2018;9:878.

31. Ramakrishna S, Kwaku Dad AB, Beloor J, Gopalappa R, Lee SK, Kim H. Gene disruption by cell-penetrating peptide-mediated delivery of Cas 9 protein and guide RNA. Genome Res. 2014:24:1020-7.

32. Park J, Bae S, Kim J-S. Cas-Designer: a web-based tool for choice of CRISPR-Cas9 target sites. Bioinformatics. 2015;31:4014-6.
33. Pohl C, Kiel JAKW, Driessen AJM, Bovenberg RAL, Nygård Y. CRISPR/ Cas9 based genome editing of Penicillium chrysogenum. ACS Synth Biol. 2016;5:754-64.

34. Zetsche B, Gootenberg JS, Abudayyeh OO, Slaymaker IM, Makarova KS, Essletzbichler P, et al. Cpf1 is a single RNA-Guided Endonuclease of a Class 2 CRISPR-Cas system. Cell. 2015;163:759-71.

35. Berka RM, Grigoriev IV, Otillar R, Salamov A, Grimwood J, Reid I, et al. Comparative genomic analysis of the thermophilic biomass-degrading fungi Myceliophthora thermophila and Thielavia terrestris. Nat Biotechnol. 2011;29:922-7

36. Zhang C, Meng X, Wei X, Lu L. Highly efficient CRISPR mutagenesis by microhomology-mediated end joining in Aspergillus fumigatus. Fungal Genet Biol. 2016;86:47-57.

37. Abdallah Q, Gel W, Fortwendel JR. A simple and universal system for gene manipulation in Aspergillus fumigatus: in vitro-assembled Cas9-guide RNA ribonucleoproteins coupled with microhomology repair templates. mSphere. 2017;2(6):1-14.

38. Katayama T, Nakamura H, Zhang Y, Pascal A, Fujii W, Maruyama Jl. Forced recycling of an AMA1-based genome-editing plasmid allows for efficient multiple gene deletion/integration in the industrial filamentous fungus Aspergillus oryzae. Appl Environ Microbiol. 2019;85:1-16.

39. Matsu-ura T, Baek M, Kwon J, Hong C. Efficient gene editing in Neurospora crassa with CRISPR technology. Fungal Biol Biotechnol. 2015;2:1-7.

40. Liu R, Chen L, Jiang Y, Zhou Z, Zou G. Efficient genome editing in filamentous fungus Trichoderma reesei using the CRISPR/Cas9 system. Cell Discov. 2015;1:15007

\section{Publisher's Note}

Springer Nature remains neutral with regard to jurisdictional claims in published maps and institutional affiliations.
Ready to submit your research? Choose BMC and benefit from:

- fast, convenient online submission

- thorough peer review by experienced researchers in your field

- rapid publication on acceptance

- support for research data, including large and complex data types

- gold Open Access which fosters wider collaboration and increased citations

- maximum visibility for your research: over $100 \mathrm{M}$ website views per year

At BMC, research is always in progress.

Learn more biomedcentral.com/submissions 